\title{
Clinical and laboratory characteristics of patients having amyloidogenic transthyretin deposition in osteoarthritic knee joints
}

\author{
Ya-jun $\mathrm{GU}^{1}$, Peng $\mathrm{GE}^{1,2}$, Yun $\mathrm{MU}^{3}$, Jin-hai LU ${ }^{1}$, Fang ZHENG ${ }^{\dagger+1}$, Xu-guo $\mathrm{SUN}^{\dagger+1}$ \\ ( ${ }^{1}$ School of Medical Laboratory, Tianjin Medical University, Tianjin 300203, China) \\ ( ${ }^{2}$ Department of Clinical Laboratory, Tianjin Medical University Cancer Institute and Hospital, Tianjin 300060, China) \\ $\left({ }^{3}\right.$ Department of Clinical Laboratory, Tianjin Children's Hospital, Tianjin 300074, China) \\ †E-mail: zf172984@aliyun.com.cn; sereg23@163.com
}

Received Feb. 9, 2013; Revision accepted July 29, 2013; Crosschecked Dec. 17, 2013

\begin{abstract}
Objective: Our aim was to investigate clinical and laboratory characteristics of osteoarthritic patients who had amyloid deposition in their knee joints. Methods: Synovial membranes were obtained from 36 patients with knee osteoarthritis $(\mathrm{OA})$ who underwent joint replacement surgery. From this sample, the diagnosis of amyloid was determined by Congo red staining, which demonstrated apple-green birefringence under a polarized microscope. All synovial membranes were immunohistochemically characterized for the expressions of amyloid immunoglobulin light chain (AL-K and AL- $\lambda$ ), serum amyloid-A (SAA), amyloidogenic transthyretin (ATTR), and amyloidogenic $\beta 2-$ microglobulin (AB2M). Matrix-assisted laser desorption-ionizaton/time of flight mass spectrometry (MALDI-TOF MS) was used to analyze transthyretin (TTR) isoforms in the serum of each patient. Results: Nine cases (25\%) were found to be amyloid-positive. Immunohistochemically, eight cases (88.9\%) had ATTR deposition, and one sample (11.1\%) was shown to be AL-K-positive. MALDI-TOF MS identified that the TTR in the serum of the patients was unmodified wild-type TTR, TTR-Cys-S-S-Cys, and TTR-Cys-S-S-CysGly. The age at surgery and the disease duration were significantly higher in the ATTR-positive group than in the ATTR-negative group. Knee score and function score were significantly lower in the ATTR-positive group than in the ATTR-negative group. Conclusions: Amyloid deposition in synovial membranes of OA patients was found to be ATTR and AL-K. TTR in the serum of the patients was unmodified wild-type TTR together with two isoforms. The high age at surgery, long disease duration, and a deteriorated knee function were associated with ATTR amyloid deposition in the osteoarthritic knee joints.
\end{abstract}

Key words: Amyloid, Immunoglobulin light chain, Matrix-assisted laser desorption-ionizaton/time of flight mass spectrometry (MALDI-TOF MS), Osteoarthritis, Transthyretin doi: 10.1631 jzus.B1300046

Document code: A

CLC number: R684.3

\section{Introduction}

Osteoarthritis (OA) is a chronic disease associated with damage to the joint cartilage and surrounding tissues and is characterized by progressive degeneration of the joints, sclerosis of the subchondral bone, and formation of new bone at the joint

\footnotetext{
${ }^{\ddagger}$ Corresponding authors

CC Zhejiang University and Springer-Verlag Berlin Heidelberg 2014
}

margins. Clinical manifestations include pain, stiffness, bony enlargement, and swelling, which ultimately lead to some degree of disability. Joint replacement surgery may be needed for severe pain or progressive joint destruction. With substantial research conducted on OA worldwide, the pathogenesis of the disease still remains uncertain. Previous reports demonstrated that inflammation in the synovial tissue may play a critical role in the pathophysiology of OA (Ray and Ray, 2008). Increased mononuclear cell 
infiltration and overexpression of proinflammatory mediators were observed in both the early and late $\mathrm{OA}$ stages (Benito et al., 2005).

Sørenson and Christensen (1973) found local amyloid deposition in the hip joint capsule in patients with OA, which was related to tissue degeneration. Egan et al. (1982) made a new observation that amyloid was often located at the articular cartilage surface of osteoarthritic knees or hips. Thereafter, Ladefoged (1983) described amyloid deposits in the articular capsule from osteoarthritic hip joints associated with secondary chondromatosis, pyrophosphate deposits, and inflammatory cell infiltration. Thus, we hypothesized that amyloidosis of the synovial tissue was a general phenomenon in patients with $\mathrm{OA}$ and was associated with progressive joint destruction. We then attempted to analyze the amyloid proteins in OA patients who underwent joint replacement surgery.

Thirty different types of amyloidosis have been described. The most frequent amyloid disorder is immunoglobulin light chain (AL) amyloidosis. It is an acquired plasma cell disease in which a monoclonal $\mathrm{AL}$ is produced in the body. Amyloid A (AA) amyloidosis is a disorder characterized by the deposition of serum amyloid A (SAA), which is associated with chronic inflammation. Hereditary (familial) amyloidosis is a diverse genetic entity, which is caused by the amyloidogenic mutations in genes encoding normal proteins such as amyloidogenic transthyretin (ATTR), apolipoprotein (AApo), A1 fibrinogen (AFib), and lysozyme (ALys). Among these, the most common form of familial amyloidosis is ATTR. However, Solomon et al. (2006) described that the amyloid in the knee menisci was formed from Apo A-I that was produced by chondrocytes within the meniscal cartilage. In the group of senile amyloidosis, wild-type ATTR was found predominantly in the tissues. Amyloidogenic $\beta 2$-microglobulin $(\mathrm{A} \beta 2 \mathrm{M})$ is a dialysis-related complication which is found in almost half of patients with hemodialysis. Hurst $e t$ al. (1989) made a survey of 95 patients with dialysisrelated arthropathy, and they found that $\mathrm{A} \beta 2 \mathrm{M}$ deposition could cause joint destruction, which indicates the possibility of $\mathrm{A} \beta 2 \mathrm{M}$ amyloidosis in osteoarthritic knee joints.

In patients with amyloid deposition, it is essential to determine the protein subtypes of the amyloid deposits. Precise identification of the amyloid subtype is imperative for appropriate treatment. Thus, the aggregation of amyloid protein in synovial membranes from patients with knee OA was analyzed in our study. Moreover, there are no previous studies assessing the relationship between patients' characteristics and the presence of amyloid in patients with knee OA. We sought to investigate the clinical and laboratory characteristics of patients having amyloid deposition in knee joints, which would be of great help to further illustrate amyloid deposition in the joint as a possible pathogenic mechanism in OA.

\section{Materials and methods}

\subsection{Patients}

Thirty-six OA patients, including 20 females and 16 males with a median age of 66 years who underwent joint replacement surgery were consecutively recruited for the study. Blood samples collected from another 36 healthy individuals were used as a normal control. The OA patients were matched with the normal controls for age and gender. Knee $\mathrm{OA}$ was diagnosed on the basis of the American College of Rheumatology (ACR) clinical classification criteria (Altman et al., 1986). Patients with other chronic diseases capable of inducing amyloidosis were excluded from the study. The knee and function scores were assessed separately according to the Knee Society Clinical Rating System (Insall et al., 1989).

\subsection{Laboratory investigation}

Prior to surgery, all patients were screened by routine laboratory tests, including blood tests and urine analysis. The C-reactive protein (CRP) and IgM rheumatoid factor (RF) were measured by enzymelinked immunosorbent assay (ELISA).

\subsection{Synovial tissue section}

Surgical specimens of synovial membranes were fixed in $10 \%$ formalin and embedded in paraffin immediately after knee joint replacement surgery. Consecutive sections of synovial membranes were used for staining with alkaline Congo red for the polarized microscopy and with monoclonal antibodies for the immunohistochemical tests. 


\subsection{Congo red staining}

Deparaffinized tissue sections $(n=36)$ were incubated for 30 min with $80 \%$ ethanol saturated with $\mathrm{NaCl}$ followed by $0.5 \%$ Congo red in $80 \%$ ethanol saturated with $\mathrm{NaCl}$. Amyloid deposition was characterized by the reddish color in Congo red staining and confirmed by apple-green birefringence under a polarized microscope.

\subsection{Immunohistochemical staining}

According to the manufacturer's instructions, immunohistochemical tests were performed on synovial tissues $(n=36)$ using monoclonal antibodies against AL- $\kappa$, AL- $\lambda$, SAA, ATTR, and A $\beta 2 \mathrm{M}$ (AssayPro, Winfield, MO, USA), respectively. Immunoreactivity was visualized with a DAB Liquid System (DAKO, Glostrup, Denmark) as described by the manufacturer.

\subsection{Immunoprecipitation and matrix-assisted laser desorption-ionizaton/time of flight mass spectrometry (MALDI-TOF MS)}

TTR was immunoprecipitated from serum of each patient and analyzed by MALDI-TOF MS as described by Tachibana et al. (1999) with minor modifications. Briefly, $500 \mu \mathrm{l}$ of serum was incubated with $30 \mu \mathrm{l}$ of rabbit anti-TTR antibody for $12 \mathrm{~h}$ at $4{ }^{\circ} \mathrm{C}$. After centrifugation at $9000 \times g$ for $15 \mathrm{~min}$, the precipitate was washed twice with $100 \mu \mathrm{l}$ of saline and twice with $100 \mu$ l of distilled water $\left(\mathrm{DH}_{2} \mathrm{O}\right)$. Next, the precipitate was dissolved in $30 \mu \mathrm{l}$ of solution containing $4 \%$ acetic acid and $4 \%$ acetonitrile. The mixture was then passed through a $1000-\mathrm{kDa}$ TTR centrifugal concentrator (Pall Filtron Northborongh, MA, USA). The pass-through fraction was mixed with $0.1 \%$ trifluoroacetic acid saturated with sinapinic acid for MALDI-TOF MS. The mass spectrometer (Bruker Franzen Analytik GmbH, Bremen, Germany) operated at a wavelength of $337 \mathrm{~nm}$. The spectrum of TTR was obtained using an ion accelerating voltage of $27.5 \mathrm{kV}$ and a reflection voltage of $30 \mathrm{kV}$. The instrument was externally calibrated using a horse myoglobin ( $m / z$ 16952.27) (Kingsbury et al., 2007).

\subsection{Statistical analysis}

The Mann-Whitney test was used to compare differences between two unpaired groups. The Wilcoxon signed-rank test was used when comparing two matched groups with a non-normal distribution or unequal variance. The correlation of the nonparametric statistics was analyzed by a Spearman rank test. For all the analyses, a two-sided $P$ value less than 0.05 was considered to be significant. Statistical analysis was performed with the software PASW statistics 18 .

\section{Results}

\subsection{General characteristics of patients with knee $\mathrm{OA}$}

Thirty-six consecutive patients (20 females and 16 males) undergoing joint replacement surgery were included in the study. The patients with amyloid did not differ in occupation, marital status, and religious background from the non-amyloid patients.

\subsection{Identification of amyloid deposition by Congo red and the polarized microscope}

Amyloid deposition was present in 9 of the 36 (25\%) synovial membranes from patients with OA. Typical apple-green birefringence was observed under the polarized microscope, which confirmed amyloid deposition in the knee joint tissues (Fig. 1). Another case was shown to be Congo red positive without the appearance of apple-green birefringence under the polarized microscope.
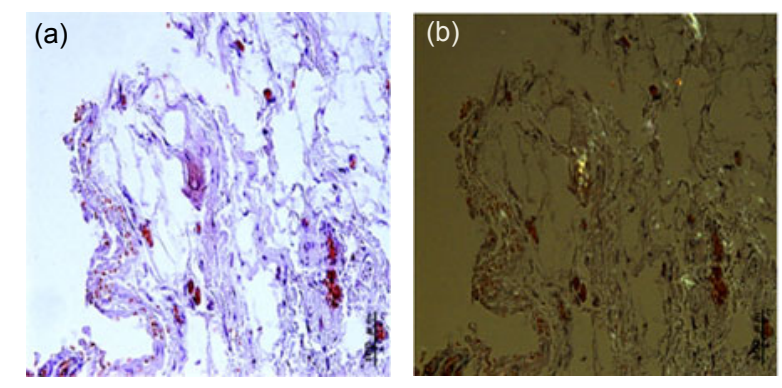

Fig. 1 Amyloid deposition in synovial membranes of $\mathrm{OA}$ patients

The reddish color of Congo red staining (a) turned into apple-green in the polarized light (b) (Note: for interpretation of the references to color in this figure legend, the reader is referred to the web version of this article)

\subsection{Analysis of amyloid subtypes by immuno- histochemistry}

Among the 36 patients with knee OA, 8 cases $(22.2 \%)$ were anti-ATTR positive and 1 sample $(2.8 \%)$ was shown to be anti-AL- $\kappa$ immunoreactive in our 
cohort (Fig. 2). There was no co-existence for both ATTR and AL- $\kappa$ in any tissue involved in the testing. On the contrary, no immunoreactivity was found using the other three antibodies directed against AL- $\lambda$, $\mathrm{SAA}$, and $\mathrm{A} \beta 2 \mathrm{M}$.

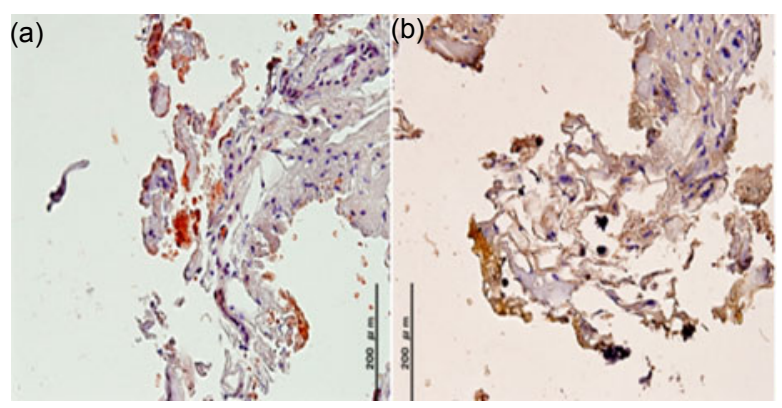

Fig. 2 Immunohistochemical results of $\mathrm{OA}$ patients (a) AL-к; (b) ATTR

\subsection{Detection of TTR isoforms by MALDI-TOF MS}

The serum of each patient $(n=36)$ was analyzed by MALDI-TOF MS. Three major peaks were observed in the mass spectrum (Fig. 3), and TTR subtypes were identical in every patient. The single peak at $13762 \mathrm{~m} / \mathrm{z}$ indicated the native, unmodified TTR. The additional peak adjacent to the unmodified TTR represented Cys adducts for $S$-cysteine (TTR-CysS-S-Cys, $13875 \mathrm{~m} / \mathrm{z}$ ). The third major peak was indicative of Cys adducts for $S$-cysteinylglycine (TTRCys-S-S-CysGly, $13932 \mathrm{~m} / \mathrm{z}$ ). No mutant TTR was found in the serum of the patients with knee OA.

\subsection{Clinical characteristics of ATTR-positive and ATTR-negative patients}

Among the nine patients with amyloid, eight cases $(88.9 \%)$ had ATTR deposits and only one sample (11.1\%) was shown to be AL-к-positive. Thus, we further analyzed the differences in clinicopathological characteristics between the ATTR-positive $(n=8)$ and ATTR-negative $(n=28)$ patients (Table 1$)$. The patients' age at surgery ranged from 58 to 72 years with a median age of 66 years. The age of patients who had ATTR deposition was significantly higher than that of the patients without ATTR-amyloid deposition $(P=0.012)$. In our series, 3 of the 16 men and 5 of the 20 women had ATTR deposition in patients with knee OA. No statistical difference was found in the gender between ATTR-positive and ATTR- negative patients. The disease duration of the ATTRpositive group was longer than that of the ATTRnegative group $(P=0.014)$. The knee and function

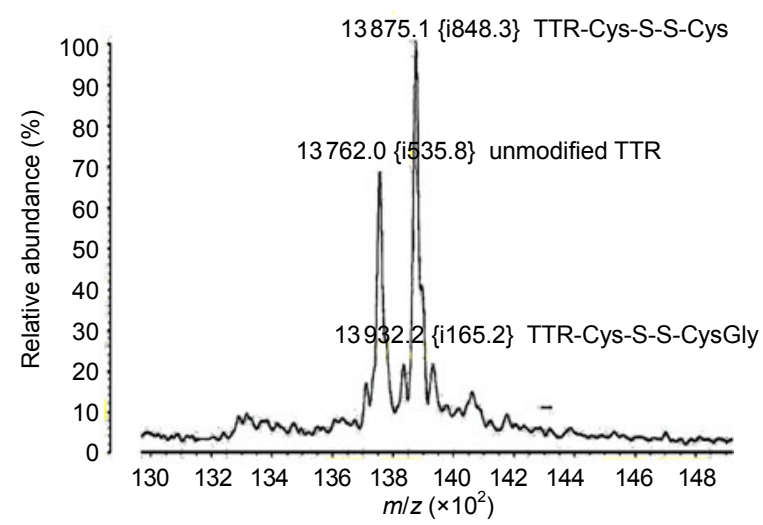

Fig. 3 Three major peaks observed in the mass spectrum, including the unmodified TTR, TTR-Cys-S-SCys, and TTR-Cys-S-S-CysGly

The proportion of each component has been associated with the peak height, measured in relative percentage $(\%)$, reflecting the relative quantity. MALDI-TOF MS showed that TTR subtypes were the same in every serum sample, whereas the proportion of unmodified TTR to total TTR was different in each patient

Table 1 Clinicopathological characteristics of the ATTRpositive and ATTR-negative patients

\begin{tabular}{lccc}
\hline \multicolumn{1}{c}{ Variable } & $\begin{array}{c}\text { ATTR-positive ATTR-negative } \\
(n=8)\end{array}$ & $P$ \\
\hline General & & & \\
Age at surgery (year) & $69.5(65-72)$ & $65.5(58-72)$ & 0.012 \\
Gender (F/M) & $5 / 3$ & $15 / 13$ & NS \\
Disease duration (year) & $16.5(7-30)$ & $12.0(5-20)$ & 0.014 \\
Clinical manifestation & & & \\
Swollen joint & 5 & 17 & NS \\
Stiffness & 5 & 19 & NS \\
Deformity & 7 & 23 & NS \\
Friction & 3 & 12 & NS \\
Knee score & $41.0(33-47)$ & $44.5(37-59)$ & 0.041 \\
Function score & $34.5(29-46)$ & $41.0(32-53)$ & 0.041 \\
Related disease & & & \\
Obesity & 1 & 3 & NA \\
Malnutrition & 1 & 4 & NA \\
Diabetes & 2 & 3 & NA \\
Drug treatment & & & \\
NSAID & 5 & 17 & NS \\
Paracetamol & 6 & 19 & NS \\
\hline Dawe shown & & & \\
\hline
\end{tabular}

Data were shown as the number of patients or median value (range). NS: not significant; NA: not applicable; NSAIDs: non-steroid antiinflammatory drugs 
scores of the ATTR-positive group were lower than those of the ATTR-negative group $(P<0.05)$. There were no statistical differences in other clinical manifestations, related diseases, drug treatment, and the presence of ATTR deposition between ATTR-positive and ATTR-negative patients.

\subsection{Laboratory measurements of ATTR and non- ATTR patients}

Erythrocyte sedimentation rate (ESR) and CRP were identified to be significantly higher in the OA patients than in the healthy individuals $(P<0.05)$ (Fig. 4). No statistical difference was found in RF, haemoglobin $(\mathrm{Hb})$, red blood cell (RBC), white blood cell (WBC), platelet, urea, uric acid, and creatinine between the OA patients and the healthy individuals. Laboratory features of the ATTR-positive and ATTRnegative patients are depicted in Table 2. No statistical difference was found in RF, ESR, CRP, $\mathrm{Hb}, \mathrm{RBC}$, WBC, platelet, urea, uric acid, or creatinine between the ATTR-positive and ATTR-negative patients.

\section{Discussion}

Amyloid diseases are caused by misfolded precursor proteins that aggregate and deposit as unique fibrils, ultimately leading to irreversible organ dysfunction. Although the role of amyloid deposition in the pathogenesis of $\mathrm{OA}$ is not clear, preventing the formation or promoting the disaggregation is necessary to control the development of clinical symptoms. Amyloid proteins usually deposit in the cartilage, articular capsule, and synovial membranes in patients with OA. Several lines of evidence demonstrated that membranes may play an essential role in protein folding, misfolding, and aggregation (Torres-Bugeau

Table 2 Laboratory measurements of the ATTR-positive and ATTR-negative patients

\begin{tabular}{|c|c|c|c|c|}
\hline Variable & $\begin{array}{c}\text { ATTR- } \\
\text { positive } \\
(n=8)\end{array}$ & $\begin{array}{c}\text { ATTR- } \\
\text { negative } \\
(n=28)\end{array}$ & $\begin{array}{c}\text { Normal } \\
\text { range }\end{array}$ & $P$ \\
\hline \multicolumn{5}{|l|}{ Blood test } \\
\hline RF (kIU/L) & $\begin{array}{c}5.5 \\
(2-21)\end{array}$ & $\begin{array}{c}6.0 \\
(2-15)\end{array}$ & $0-10$ & NS \\
\hline $\operatorname{ESR}(\mathrm{mm} / \mathrm{h})$ & $\begin{array}{c}27.0 \\
(15-52)\end{array}$ & $\begin{array}{c}24.5 \\
(1-46)\end{array}$ & $0-30$ & NS \\
\hline $\mathrm{CRP}(\mathrm{mg} / \mathrm{L})$ & $\begin{array}{c}2.4 \\
(1.2-5.7)\end{array}$ & $\begin{array}{c}2.2 \\
(1.3-4.3)\end{array}$ & $0-2.3$ & NS \\
\hline $\mathrm{Hb}(\mathrm{g} / \mathrm{L})$ & $\begin{array}{c}126.5 \\
(72-151)\end{array}$ & $\begin{array}{c}116.5 \\
(83-158)\end{array}$ & $110-160$ & NS \\
\hline $\mathrm{RBC}\left(\times 10^{12} / \mathrm{L}\right)$ & $\begin{array}{c}4.2 \\
(2.8-5.8)\end{array}$ & $\begin{array}{c}4.3 \\
(3.0-5.8)\end{array}$ & $3.5-5.5$ & NS \\
\hline $\mathrm{WBC}\left(\times 10^{9} / \mathrm{L}\right)$ & $\begin{array}{c}9.0 \\
(4.7-13.6)\end{array}$ & $\begin{array}{c}7.4 \\
(3.8-12.9)\end{array}$ & $4-10$ & NS \\
\hline Platelet $\left(\times 10^{9} / \mathrm{L}\right)$ & $\begin{array}{c}248.5 \\
(204-429)\end{array}$ & $\begin{array}{c}236.5 \\
(98-401)\end{array}$ & $100-300$ & NS \\
\hline Urea $(\mathrm{mmol} / \mathrm{L})$ & $\begin{array}{c}4.4 \\
(2.9-8.1)\end{array}$ & $\begin{array}{c}4.8 \\
(2.3-7.2)\end{array}$ & $2.5-6.4$ & NS \\
\hline Uric acid $(\mu \mathrm{mol} / \mathrm{L})$ & $\begin{array}{c}267.5 \\
(167-510)\end{array}$ & $\begin{array}{c}278.0 \\
(178-489)\end{array}$ & $150-400$ & NS \\
\hline Creatinine $(\mu \mathrm{mol} / \mathrm{L})$ & $\begin{array}{c}81.5 \\
(58-167)\end{array}$ & $\begin{array}{c}67.5 \\
(44-172)\end{array}$ & $40-120$ & NS \\
\hline \multicolumn{5}{|l|}{ Urine analysis } \\
\hline Proteinuria & 2 & 3 & & NA \\
\hline Pyuria & 1 & 2 & & NA \\
\hline Hematuria & 1 & 2 & & NA \\
\hline
\end{tabular}

Data were shown as the number of patients or median value (range). NS: not significant; NA: not applicable; RF: rheumatoid factor; ESR: erythrocyte sedimentation rate; CRP: C-reactive protein; $\mathrm{Hb}$ : haemoglobin; RBC: red blood cell; WBC: white blood cell (a)

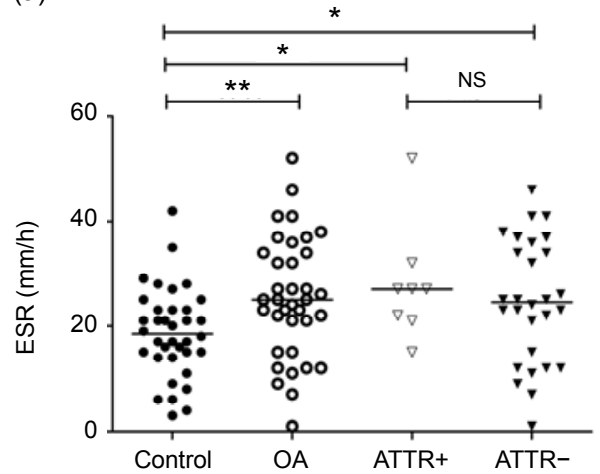

(b)

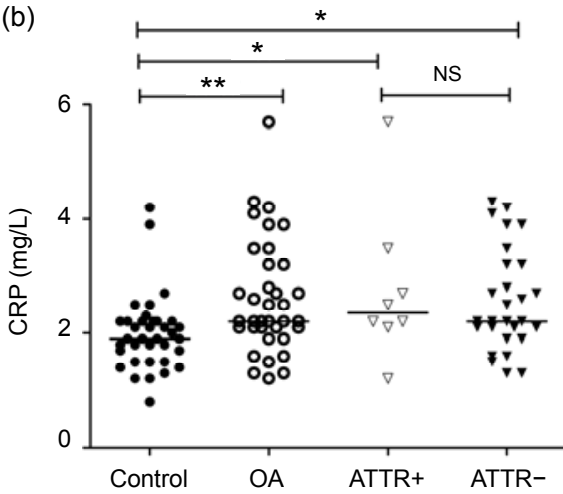

Fig. 4 Comparsion of the ATTR-positive and ATTR-negative patients

(a) Erythrocyte sedimentation rate (ESR); (b) The C-reactive protein (CRP). Horizontal lines indicated median levels and the asterisks indicated the differences between two groups. ${ }^{*} P<0.05 ;{ }^{* *} P<0.01$; NS: not significant 
et al., 2011). Thus, synovial membranes were selected for this study. Amyloid deposition has previously been reported in $33 \%$ of OA patients who underwent total reconstruction of the hip joint (Christensen and Sørenson, 1972). In these patients, the amyloid was primarily situated in the articular capsule of the hip. Amyloid deposits were present in 9 of $36(25 \%)$ synovial membranes for patients with knee OA in our study. Another case was shown to be Congo red positive without the appearance of apple-green birefringence under the polarized microscope. This is probably a non-specific staining phenomenon without any significance. Amyloid infiltration into synovial membranes may accelerate the inflammatory process and osteolytic responses.

Around 30 different amyloid precursor proteins have been identified to result in either local tissue or systemic amyloidosis (Sipe et al., 2012). In our study, all synovial membranes were analyzed by Congo red staining and immunohistochemistry, respectively. Nine cases were found to be amyloid-positive by Congo red staining, which demonstrated apple-green birefringence under the polarized microscope. Moreover, the same cases $(n=9)$ were found to be immunohistochemically positive, including ATTR $(n=8)$ and AL- $\kappa(n=1)$. Because the number of amyloidpositive cases was equal to that of the immunohistochemically positive specimens, we did not look for other rare amyloid types. We think that amyloid in the knee OA has been derived from ATTR and AL- $\kappa$ without co-expression of rare types. However, Solomon et al. (2006) demonstrated that the amyloid proteins in knee menisci were derived from Apo A1 and not from ATTR. In their study, amyloid was extracted from the menisci of three adults who underwent knee joint replacement surgery. In our experiment, synovial membranes were used to analyze amyloid deposition. Thus, we hypothesize that amyloid deposits may differ in selected samples of patients with knee OA. More case studies may be needed to validate this claim. The frequency of $\mathrm{AL}$ amyloid arthropathy has been estimated to range from $2 \%$ to $5 \%$ (M'Bappé and Grateau, 2012). Similarly, one case $(2.8 \%)$ was shown to be anti-AL-א immunoreactive in our studies. Niggemeyer et al. (2011) found ATTR deposition as the only immunohistological subtype in patients with the hip OA. Our study confirmed previous reports in which amyloid deposition was observed in OA patients, especially AL and ATTR deposition.

TTR is a serum and cerebrospinal fluid transport protein for thyroxine and retinol binding protein, which is known to be associated with amyloidosis. The wild-type TTR is the only fibrillar protein, which contributes to the development of senile systemic amyloidosis with amyloid deposition in the heart, lungs, and gastrointestinal tract (Gorevic et al., 1989). Mutant TTR in relation to familial amyloidotic polyneuropathy (FAP) was found to be more prone to form amyloid, especially in the heart and nerves (Ihse et al., 2011). In our study, the amyloid deposition in synovial membranes of patients with knee OA was found to be wild-type TTR without mutations. Moreover, the MALDI-TOF MS analysis of serum showed that TTR subtypes were identical in every patient (unmodified wild-type together with two isoforms). Post-translational modification of TTR may play a role in its pathogenicity in amyloidosis.

Among the nine patients with amyloid, eight cases $(88.9 \%)$ had ATTR deposition, only one tissue section $(11.1 \%)$ was shown to be AL-א-positive. Thus, we further analyzed the differences in clinical characteristics between the ATTR-positive and ATTRnegative patients. Some statistical differences in patients' characteristics were found between them, including age at surgery, disease duration, knee score, and function score. Other clinical and laboratory parameters could not predict the development of amyloid in our study. However, they should not be ignored as potential risk factors for developing amyloid in the knee OA. Further prospective studies with larger case numbers may be needed to clarify this issue.

Hattori et al. (2003) demonstrated that the frequency of amyloid increased with patients' age. Similarly, the age of patients who had ATTR deposition was significantly higher than that of the patients without amyloid deposition, which indicated that aging may play an important role in the development of ATTR deposition of the knee OA. The frequency of AA amyloid is $7 \%$ in the group of arthritis patients with 5 years or more duration, and increases to $16 \%$ in the group with disease duration of more than 10 years (El Mansoury et al., 2002). In our cohort, the prevalence of ATTR deposition is $12.5 \%$ in the group of OA patients with 10 years or less duration, and increases to $87.5 \%$ in the group with disease duration of 
more than 10 years. However, no statistical correlation was found between the age at surgery and the disease duration.

Deposition of ATTR in knee joints may weaken the synovial membranes, resulting in local inflammation followed by progressive degeneration of the joints. Cortez et al. (2010) demonstrated that membranes had the ability to shift the protein folding equilibrium with different structural features towards the aggregation state. However, stiffness, deformity, friction, or swollen joints cannot be individually used to predict the presence of ATTR deposition in our study. We found significant differences in the knee and function scores between the ATTR-positive and ATTR-negative groups, which indicated that amyloid infiltration into synovial membranes might play a role in multiple pathogenic mechanisms of OA. According to the report of Kuroda et al. (2002), the patients with knee OA showed laboratory signs of inflammation with significantly increased serum levels of ESR and CRP. Though ESR and CRP were identified to be significantly higher in the OA patients than in the healthy individuals, no difference was found in ESR or CRP between the ATTR-positive and ATTRnegative groups in our study.

In summary, amyloid deposition in synovial membranes of OA patients was found to be ATTR and AL- $\kappa$. The TTR in the serum of the patients was unmodified wild-type TTR together with two isoforms (unmodified TTR, TTR-Cys-S-S-Cys, TTR-Cys-S-SCysGly). High age at surgery, long disease duration, and deteriorated knee function were associated with ATTR deposition in the osteoarthritic knee joints. Amyloid infiltration into synovial membranes may play a role in pathogenicity of amyloidosis in OA patients, which needs to be further explored in more case studies.

\section{Compliance with ethics guidelines}

Ya-jun GU, Peng GE, Yun MU, Jin-hai LU, Fang ZHENG, and $\mathrm{Xu}$-guo SUN declare that they have no conflict of interest.

All procedures followed were in accordance with the ethical standards of the responsible committee on human experimentation (institutional and national) and with the Helsinki Declaration of 1975, as revised in 2008 (5). Informed consent was obtained from all patients for being included in the study. Additional informed consent was obtained from all patients for which identifying information is included in this article.

\section{References}

Altman, R., Asch, E., Bloch, D., et al., 1986. Development of criteria for the classification and reporting of osteoarthritis. Classification of osteoarthritis of the knee. Arthritis Rheum., 29(8):1039-1049. [doi:10.1002/art.1780290816]

Benito, M.J., Veale, D.J., FitzGerald, O., et al., 2005. Synovial tissue inflammation in early and late osteoarthritis. Ann. Rheum. Dis., 64(9):1263-1267. [doi:10.1136/ard.2004. 025270]

Christensen, H.E., Sørenson, K.H., 1972. Local amyloid formation of capsula fibrosa in arthrosis coxae. Acta Pathol. Microbiol. Scand. Suppl., 233:128-131.

Cortez, L.M., Ávila, C.L., Torres Bugeau, C.M., et al., 2010. Glyceraldehyde-3-phosphate dehydrogenase tetramer dissociation and amyloid fibril formation induced by negatively charged membranes. FEBS Lett., 584(3):625630. [doi:10.1016/j.febslet.2009.12.012]

Egan, M.S., Goldenberg, D.L., Cohen, A.S., et al., 1982. The association of amyloid deposits and osteoarthritis. Arthritis Rheum., 25(2):204-208. [doi:10.1002/art.17802 50214]

El Mansoury, T.M., Hazenberg, B.P.C., El Badawy, S.A., et al., 2002. Screening for amyloid in subcutaneous fat tissue of Egyptian patients with rheumatoid arthritis: clinical and laboratory characteristics. Ann. Rheum. Dis., 61(1):42-47. [doi:10.1136/ard.61.1.42]

Gorevic, P.D., Prelli, F.C., Wright, J., et al., 1989. Systemic senile amyloidosis. Identification of a new prealbumin (transthyretin) variant in cardiac tissue: immunologic and biochemical similarity to one form of familial amyloidotic polyneuropathy. J. Clin. Invest., 83(3):836-843. [doi:10.1172/JCI113966]

Hattori, T., Takei, Y., Koyama, J., et al., 2003. Clinical and pathological studies of cardiac amyloidosis in transthyretin type familial amyloid polyneuropathy. Amyloid, 10(4):229-239. [doi:10.3109/13506120309041740]

Hurst, N.P., van den Berg, R., Disney, A., et al., 1989. 'Dialysis related arthropathy': a survey of 95 patients receiving chronic haemodialysis with special reference to $\beta_{2}$ microglobulin related amyloidosis. Ann. Rheum. Dis., 48(5):409-420. [doi:10.1136/ard.48.5.409]

Ihse, E., Suhr, O.B., Hellman, U., et al., 2011. Variation in amount of wild-type transthyretin in different fibril and tissue types in ATTR amyloidosis. J. Mol. Med., 89(2): 171-180. [doi:10.1007/s00109-010-0695-1]

Insall, J.N., Dorr, L.D., Scott, R.D., et al., 1989. Rationale of the Knee Society clinical rating system. Clin. Orthop. Relat. Res., 248:13-14.

Kingsbury, J.S., Klimtchuk, E.S., Théberge, R., et al., 2007. Expression, purification, and in vitro cysteine-10 modification of native sequence recombinant human transthyretin. Protein Express. Purif., 53(2):370-377. [doi:10. 1016/j.pep.2007.01.004]

Kuroda, T., Tanabe, N., Sakatsume, M., et al., 2002. Comparison of gastroduodenal, renal and abdominal fat 
biopsies for diagnosing amyloidosis in rheumatoid arthritis. Clin. Rheumatol., 21(2):123-128. [doi:10.1007/ PL00011217]

Ladefoged, C., 1983. Amyloid in osteoarthritic hip joints: deposits in relation to chondromatosis, pyrophosphate, and inflammatory cell infiltrate in the synovial membrane and fibrous capsule. Ann. Rheum. Dis., 42(6):659-664. [doi:10.1136/ard.42.6.659]

M'Bappé, P., Grateau, G., 2012. Osteo-articular manifestations of amyloidosis. Best Pract. Res. Clin. Rheumatol., 26(4): 459-475. [doi:10.1016/j.berh.2012.07.003]

Niggemeyer, O., Steinhagen, J., Deuretzbacher, G., et al., 2011. Amyloid deposition in osteoarthritis of the hip. Arch. Orthop. Trauma Surg., 131(5):637-643. [doi:10.1007/ s00402-010-1187-z]

Ray, A., Ray, B.K., 2008. An inflammation-responsive transcription factor in the pathophysiology of osteoarthritis. Biorheology, 45(3-4):399-409. [doi:10.3233/BIR-20080500]

Sipe, J.D., Benson, M.D., Buxbaum, J.N., et al., 2012. Amyloid fibril protein nomenclature: 2012 recommendations from the Nomenclature Committee of the International Society of Amyloidosis. Amyloid, 19(4):167-170. [doi:10. 3109/13506129.2012.734345]

Solomon, A., Murphy, C.L., Kestler, D., et al., 2006. Amyloid contained in the knee joint meniscus is formed from apolipoprotein A-I. Arthritis Rheum., 54(11):3545-3550. [doi:10.1002/art.22201]

Sørenson, K.H., Christensen, H.E., 1973. Local amyloid formation in the hip joint capsule in osteoarthritis. Acta Orthop. Scand., 44(4):460-466.

Tachibana, N., Tokuda, T., Yoshida, K., et al., 1999. Usefulness of MALDUTOF mass spectrometry of immunoprecipitated serum variant transthyretin in the diagnosis of familial amyloid polyneuropathy. Amyloid, 6(4):282-288. [doi:10.3109/13506129909007341]

Torres-Bugeau, C.M., Borsarelli, C.D., Minahk, C.J., et al., 2011. The key role of membranes in amyloid formation from a biophysical perspective. Curr. Protein Pept. Sci., 12(3):166-180. [doi:10.2174/138920311795860197]

\section{中文概要:}

\section{本文题目：伴发 ATTR 型淀粉样沉积的骨关节炎患者的临床和生物学特征分析}

Clinical and laboratory characteristics of patients having amyloidogenic transthyretin deposition in osteoarthritic knee joints

研究目的: 旨在检测骨关节炎患者是否存在淀粉样变, 分析淀粉样沉积的类型, 并进一步研究与淀粉 样沉积相关的临床和生物学指标。

创新要点: 首次发现了骨关节炎淀粉样沉积相关的临床和生物学指标, 为研究骨关节炎的发生机制提 供了科学依据。

研究方法: 收集 36 例接受关节置换术的骨关节炎患者的滑膜组织, 利用刚果红染色结合偏振光显微镜 鉴定滑膜组织是否发生淀粉样变沉积, 并用免疫组化染色确定淀粉样变沉积蛋白的类型。 最后用基质辅助激光解析电离飞行时间质谱检测血液中 TTR 基因突变的类型。

重要结论: 在 36 例接受关节置换术的骨关节炎患者中, 9 例 $(25 \%$ ) 出现了淀粉样沉积, 其中 8 例为 ATTR 相关的淀粉样变, 1 例与 AL- $\kappa$ 相关。基质辅助激光解析电离飞行时间质谱显示 (见 图 3), ATTR 为野生型和修饰型蛋白, 未发现突变的 ATTR 亚型。患者年龄和病程与 ATTR 淀粉样变呈正相关, 膝关节评分和功能评分与 ATTR 淀粉样变呈负相关。因此, 高龄、长 病程、膝关节功能较差的骨关节炎患者可能同时伴发 ATTR 淀粉样沉积。在常规诊疗的同 时, 对于该类患者应给予足够重视, 适当开展个体化治疗。

关键词组: 免疫球蛋白轻链; 质谱; 骨关节炎; 淀粉样变; 转甲状腺素蛋白 (ATTR) 